\title{
Minute ventilation/carbon dioxide production in patients with dysfunctional breathing
}

\author{
Matthew Watson ${ }^{1}$, Maria Florina Ionescu ${ }^{1}$, Karl Sylvester ${ }^{2,3}$ and Jonathan Fuld ${ }^{4}$ \\ Number 3 in the Series "Ventilatory efficiency and its clinical prognostic value in cardiorespiratory disorders" \\ Edited by Pierantonio Laveneziana and Paolo Palange
}

\author{
${ }^{1}$ School of Clinical Medicine, University of Cambridge, Cambridge, UK. ${ }^{2}$ Lung Function, Cambridge University Hospitals NHS \\ Foundation Trust, Cambridge, UK. ${ }^{3}$ Respiratory Physiology, Papworth Hospitals NHS Foundation Trust, Cambridge, UK. ${ }^{4}$ Dept of \\ Respiratory Medicine, Cambridge University Hospitals NHS Foundation Trust, Cambridge, UK.
}

Corresponding author: Matthew Watson (mw696@cam.ac.uk)

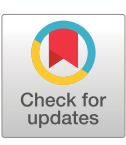

Copyright OERS 2021.

This article is open access and distributed under the terms of the Creative Commons Attribution Non-Commercial Licence 4.0.

Received: 6 June 2020 Accepted: 11 July 2020

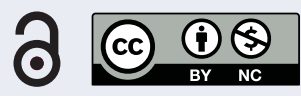

Shareable abstract (@ERSpublications)

Ventilatory equivalent for $\mathrm{CO}_{2}$ can provide information to aid the diagnosis of dysfunctional breathing, particularly hyperventilation syndrome https://bit.ly/2OwTMqY

Cite this article as: Watson M, Ionescu MF, Sylvester $\mathrm{K}$, et al. Minute ventilation/carbon dioxide production in patients with dysfunctional breathing. Eur Respir Rev 2021; 30: 200182 [DOI: 10.1183/ 16000617.0182-2020].

\section{Abstract}

Dysfunctional breathing refers to a multi-dimensional condition that is characterised by pathological changes in an individual's breathing. These changes lead to a feeling of breathlessness and include alterations in the biomechanical, psychological and physiological aspects of breathing. This makes dysfunctional breathing a hard condition to diagnose, given the diversity of aspects that contribute to the feeling of breathlessness. The disorder can debilitate individuals without any health problems, but may also be present in those with underlying cardiopulmonary co-morbidities. The ventilatory equivalent for $\mathrm{CO}_{2}$ $\left(V_{\text {eqCO }}\right)$ is a physiological parameter that can be measured using cardiopulmonary exercise testing. This review will explore how this single measurement can be used to aid the diagnosis of dysfunctional breathing. A background discussion about dysfunctional breathing will allow readers to comprehend its multidimensional aspects. This will then allow readers to understand how $V_{\text {eqCO}}$ can be used in the wider diagnosis of dysfunctional breathing. Whilst $V_{\mathrm{eqCO}}$ cannot be used as a singular parameter in the diagnosis of dysfunctional breathing, this review supports its use within a broader algorithm to detect physiological abnormalities in patients with dysfunctional breathing. This will allow for more individuals to be accurately diagnosed and appropriately managed.

\section{Introduction}

Dysfunctional breathing is a multi-dimensional condition that describes a broad range of abnormalities in an individual's breathing pattern that leads to the feeling of breathlessness. It is a condition that severely impacts the health-related quality of life of individuals [1], but can be improved with certain breathing exercises [2]. The prevalence of dysfunctional breathing is hard to estimate given how difficult it is to diagnose. However, it has been shown that the overall estimate of individuals' experiencing symptoms characteristic of dysfunctional breathing is between $8 \%$ and $9.5 \%$ in the general population [3]. Therefore, improving the diagnosis and management of this condition will improve the lives of many people.

Many authors have proposed classification systems to allow categorisation of dysfunctional breathing [4-6]. Dysfunctional breathing is a multifaceted term that encompasses changes in the biomechanical, psychological and physiological aspects of breathing. Alterations in each of these aspects of breathing can occur to varying degrees in patients, leading to different types of dysfunctional breathing.

A physiological response that can be utilised in the assessment of dysfunctional breathing is the relationship between minute ventilation $\left(V_{\mathrm{E}}^{\prime}\right)$ and carbon dioxide production $\left(V_{\mathrm{CO}_{2}}^{\prime}\right)$ and is often presented 
as $V_{\mathrm{E}}^{\prime} / V^{\prime} \mathrm{CO}_{2}$. However, manuscripts have often confused the ventilatory slope, which is $V_{\mathrm{E}}^{\prime} / V^{\prime} \mathrm{CO}_{2}$, with the ventilatory equivalents, which should be displayed as $V_{\text {eqCO }}$ (ventilatory equivalent for $\mathrm{CO}_{2}$ ) [7]. More in depth descriptions and comparisons between these two variables are discussed later.

It has recently been stated that there is no gold standard diagnostic procedure to diagnose dysfunctional breathing [6]. Thus, after outlining our understanding of dysfunctional breathing and the current diagnostic methods available, this review will determine the value of $V_{\mathrm{eqCO}}$ in a diagnostic algorithm for dysfunctional breathing. It should be noted that this review is not proposing $V_{\mathrm{eqCO}}$ be used as the only determinant as to whether someone has dysfunctional breathing, but rather used in conjunction with other diagnostic methods.

\section{Dysfunctional breathing}

Pathophysiology of dysfunctional breathing

Dysfunctional breathing is a broad term for conditions where alterations in the breathing pattern gives rise to dyspnoea and associated non-respiratory symptoms. The symptoms experienced are often precipitated by physiological or psychological stress, such as illness, excessive aerobic training and bereavement [8, 9]. Underlying respiratory diseases, musculoskeletal dysfunction or altered chest wall shapes can contribute to the development of the pathological breathing pattern [5]. As such, the prevalence of dysfunctional breathing is significantly higher in patients with an underlying respiratory disorder, such as COPD, than the general population [3].

The most important identifying criterion is the presence of breathlessness once organic disease has been ruled out or optimised by treatment. This concept of "disproportionate breathlessness" compared to the severity of underlying disease, if any, is key to understanding and identifying cases of dysfunctional breathing $[5,10]$.

The development of dysfunctional breathing is thought to be an unconsciously learned habitual change from the normal pattern of breathing, which may begin as a "coping mechanism" to deal with periods of stress. In these times of stress, it has been observed that the diaphragm flattens and becomes less mobile; thus, the accessory and intercostal muscles contribute more to movement required for breathing [8, 9]. This makes the breathing less efficient and fatiguing of these muscles explains some of the symptoms these patients experience: chest pain and dyspnoea. Additionally, these changes are associated with changes in the patterning of breaths; patients exhibit erratic rates and volumes of respiration with rapid shallow breaths or periodic deep sighing breaths. An increase in respiration rate may be observed, sometimes resulting in measurable hypocapnia [5].

However, the exact patterning of breathing is not always the same, and there have been attempts to classify dysfunctional breathing into subtypes [4, 5]. BARKER and EvERARD [5] split dysfunctional breathing into thoracic and extra-thoracic forms, and within these two groups, structural and functional subtypes. This paper will focus on functional thoracic dysfunctional breathing. Boulding et al. [4] proposed a further classification system for subtypes of dysfunctional breathing based on the pattern on pathophysiology. They proposed the following subtypes: hyperventilation, periodic deep sighing, thoracic dominant breathing, forced abdominal expiration and thoraco-abdominal asynchrony. These forms would all fall under the functional thoracic classification of BARKER and EvERARD [5]. However, it is important to recognise their differing pathophysiology, which gives rise to different erratic patterns of breathing. These different patterns of breathing lead to different changes in the biomechanical, psychological and physiological aspects of breathing in each subtype. The biomechanical aspects refer to how the chest wall moves and produces the pressures required for ventilation. Pathological changes in this aspect of breathing includes asynchrony between the muscles, chest wall and abdomen. The psychological aspects of dysfunctional breathing includes how an individual's mental state can affect their breathing pattern, e.g. anxiety can cause individuals to hyperventilate. Physiological aspects of breathing include changes in the ventilation relative to the amount of carbon dioxide produced, indicated by the $V_{\mathrm{eqCO}}$ and $V_{\mathrm{E}}^{\prime} / V^{\prime} \mathrm{CO}_{2}$ slope.

An example of how these different aspects of different subtypes of dysfunctional breathing interact can be demonstrated by comparing thoraco-abdominal asynchrony and hyperventilation syndrome [4]. Thoraco-abdominal asynchrony is a type of dysfunctional breathing that is characterised by the lack of synchronous movements between the abdominal wall and thoracic cage, that leads to a feeling of breathlessness. Thoraco-abdominal asynchrony therefore describes a predominantly biomechanical problem with an individual's breathing pattern. However, hyperventilation syndrome is another type of dysfunctional breathing that is instead characterised by respiratory alkalosis and an increased respiratory rate. This can also lead to the feeling of breathlessness, but is predominantly associated with psychological 
(increased anxiety leading to changes in breathing pattern) and physiological (raised $V_{\mathrm{eqCO}_{2}}$ ) changes in an individual's breathing that may not be seen in thoraco-abdominal asynchrony. It is important to understand that the term "dysfunctional breathing" encompasses a wide variety of abnormalities in an individual's breathing, which all lead to the feeling of breathlessness.

Furthermore, the various classifications of dysfunctional breathing are not discrete. Patients with hyperventilation syndrome may also have some pathological biomechanical aspects to their dysfunctional breathing [11]. Thus, it would be difficult to effectively diagnose dysfunctional breathing by observing just one aspect of an individual's breathing.

\section{Diagnostic tools used for dysfunctional breathing}

Despite many diagnostic methods in clinical use, dysfunctional breathing remains difficult to diagnose, without clear overlap between diagnoses picked up by the different methods [12]. This is partly due to a lack of consensus as to the exact definition and classification of dysfunctional breathing. Another difficulty arises from the co-incidence and symptom overlap with other respiratory conditions; dysfunctional breathing co-existing with COPD may be missed and symptoms simply labelled "severe COPD”. A further complicating factor, particularly for physical or observation assessment methods, is that the dysfunctional breathing pattern may not be manifested all the time. It has also been discussed how different types of dysfunctional breathing can have different changes in the biomechanical, physiological and psychological features of breathing. Thus, to effectively diagnose dysfunctional breathing, there needs to be a range of diagnostic tests used to characterise these facets of breathing. The diagnostic tests used will be discussed in relation to identifying abnormalities in the psychological, biomedical and physiological changes that occur in dysfunctional breathing.

Questionnaire-based approaches, such as the Nijmegen Questionnaire and the self-evaluation of breathing questionnaire (SEBQ) [13] are commonly used in clinical practice to diagnose dysfunctional breathing. These questionnaires are limited by their degree of subjectivity since patients are asked to quantify their own symptoms; they may not necessarily notice their own symptoms or report their frequency accurately. These questionnaires highlight the patient's perceptions of their own breathing, thus giving a good indication to whether there is a psychological drive to the dysfunctional breathing.

The Nijmegen Questionnaire is the most widespread method used in the clinical diagnosis of dysfunctional breathing. It is comprised of a series of questions that assess how frequently the patient experiences certain symptoms associated with hyperventilation. The Nijmegen questionnaire was found to have a high specificity of $95 \%$ and sensitivity of $91 \%$ in detecting hyperventilation [14]. However, in the context of patients with other conditions, such as asthma, COPD, panic disorder and anxiety, the specificity is lower, since the Nijmegen Questionnaire cannot differentiate symptoms caused by the dysfunctional breathing and those from the underlying co-morbidity $[15,16]$. It has been suggested that the Nijmegen questionnaire score represents a subjective score of "functional respiratory complaints" that may only be indicative of hyperventilation or other forms of dysfunctional breathing [15].

Other common methods to diagnose dysfunctional breathing in clinical practice use observation and examination by expert chest physiotherapists. These diagnostic techniques include the breathing pattern assessment tool (BPAT) [12] and manual assessment of respiratory motion (MARM) [17]. The benefit of these assessment methods is that, rather than relying on patient-reported symptom frequency, they utilise assessment by an observer. Physiotherapists can identify dysfunctional breathing using pattern recognition of visual or physical cues. These techniques can allow characterisation of the biomechanical facets of breathing, given the synchrony of breathing is being examined here.

Respiratory inductance plethysmography can also be used to diagnose dysfunctional breathing, albeit rarely used in clinical practice. Plethysmography measures the changes in volume of the chest wall, ribcage and abdomen to assess the underlying breathing mechanics. They are currently more commonly used in research scenarios, but the literature does provide support for plethysmography as a useful tool to characterise breathing patterns [18]. They could potentially be useful to characterise thoracic-dominant and thoraco-abdominal asynchronous subtypes of dysfunctional breathing.

Cardiopulmonary exercise testing (CPET) is a tool that can be used to diagnose dyspnoea of unknown cause [19-24]. CPET begins with a resting phase analysis and the subject being asked to quantify their baseline level of breathlessness and muscle fatigue to assess their usual functional status. The estimate of their functional status aids in creating the predicted workload ramp rate to use during the test, so that the subject's maximal exertion point is reached 8-12 mins into loaded cycling. Then the test proceeds to 
unloaded cycling for 3 mins, and subsequently loaded cycling, on a stationary exercise bike. Throughout this exercise, the physiological responses to the increasing work are recorded. During loaded cycling, the work rate incrementally increases until the patient is stopped by symptoms or the clinician stops the test due to safety concerns. At this peak exercise point, the patient is again asked to quantify their feelings of breathlessness and muscle fatigue, to help establish a limiting factor. In patients with dysfunctional breathing, feelings of breathlessness or air hunger are typical factors that lead to exercise cessation. The analysis of the physiological parameters continues through the recovery period until the patient has returned to their baseline at rest.

CPET provides a comprehensive overview of many physiological parameters both at rest, upon exertion and during the recovery period. Aerobic, ventilatory, cardiac, gas exchange and muscle response to exercise can be analysed and compared to normal physiological responses [25]. This is an objective method in diagnosing dysfunctional breathing, since it can both rule out other pathophysiological causes of dyspnoea and look in detail at erratic ventilation patterns. Particularly useful for the diagnosis of dysfunctional breathing are the respiratory parameters CPET measures: $V_{\mathrm{E}}^{\prime} / V_{\mathrm{CO}_{2}}^{\prime}$ slope, $V_{\mathrm{eqCO}}$, the respiratory exchange ratio, end-tidal carbon dioxide tension, respiratory rate and tidal volume $\left(\mathrm{V}_{\mathrm{T}}\right)$. Analysis of these parameters together can provide an overall picture of the subject's breathing pattern and provide an indication of whether dysfunctional breathing is present. The main limitation of CPET currently is that there is no specific criteria or thresholds for any of these parameters that can provide a definite indication of dysfunctional breathing; the diagnosis remains an inference from analysing the various data plots and parameters. In this review, the $V_{\mathrm{eqCO}}$ will be focussed on, looking particularly at the evidence as to how this parameter can aid the diagnosis of dysfunctional breathing.

\section{Can $V^{\prime}{ }_{E} / V^{\prime}{ }_{\mathrm{CO}}$ be used to aid the diagnosis of dysfunctional breathing?}

Methods used to express $\mathrm{V}^{\prime}{ }_{E} \mathrm{~N}^{\prime} \mathrm{CO}_{2}$

$V^{\prime}{ }_{E} / V^{\prime} \mathrm{CO}_{2}$ can refer to two different parameters commonly measured using CPET. Both will be discussed, allowing the reader to have a sound understanding of the descriptions and conclusions made in the next section of the manuscript. Table 1 provides readers with definitions of terms and equations used.

The first method involves plotting a $V_{E}^{\prime} / V^{\prime} \mathrm{CO}_{2}$ slope. $V_{\mathrm{E}}^{\prime}$ is plotted on the y-axis against the $V^{\prime} \mathrm{CO}_{2}$ on the $\mathrm{x}$-axis. As exercise intensity increases, there is an increase in the rate of metabolism. This results in an

\section{TABLE 1 Definitions of terms and equations used}

\section{Abbreviation}

Physiological parameter

\begin{tabular}{|c|c|c|}
\hline $\mathrm{PaCO}_{2}$ & $\begin{array}{l}\text { Arterial carbon dioxide tension } \\
\qquad(\mathrm{kPa})\end{array}$ & Reflective of the concentration of $\mathrm{CO}_{2}$ in the arterial blood \\
\hline d & Dead space volume (L) & Volume of air that is inhaled that does not take part in gas exchange \\
\hline$V_{\mathrm{T}}$ & Tidal volume (L) & Volume of air moved into and out of the lungs during each ventilation cycle \\
\hline$V_{\mathrm{E}}^{\prime}$ & Minute ventilation $\left(\mathrm{L} \cdot \mathrm{min}^{-1}\right)$ & Volume of gas exhaled/inhaled in $1 \mathrm{~min}$, equal to the $V_{\mathrm{T}}$ multiplied by the RR \\
\hline$V^{\prime} \mathrm{CO}_{2}$ & $\begin{array}{l}\text { Carbon dioxide production } \\
\left(\mathrm{L} \cdot \mathrm{min}^{-1}\right)\end{array}$ & \\
\hline $\mathrm{d} / V_{\mathrm{T}}$ & $\begin{array}{l}\text { Ratio of dead space volume to tidal } \\
\text { volume }\end{array}$ & $\begin{array}{c}\text { A higher value indicates a larger proportion of air not engaging in gas exchange within the } \\
\text { lungs }\end{array}$ \\
\hline$V_{\mathrm{E}}^{\prime} / V^{\prime} \mathrm{CO}_{2}$ & Gradient of the $V_{E}^{\prime} / V^{\prime} \mathrm{CO}_{2}$ slope & The minute ventilation required to exhale $1 \mathrm{~L}$ of $\mathrm{CO}_{2}$ \\
\hline & & Equation $\mathrm{A}: \frac{V_{\mathrm{E}}^{\prime}}{V_{\mathrm{CO}_{2}}^{\prime}}=\frac{863}{\left[P_{\mathrm{aCO}_{2}}\left(1-\frac{d}{V_{\mathrm{T}}}\right)\right]}$ \\
\hline RR & Respiratory rate $\left(\mathrm{min}^{-1}\right)$ & Number of breaths taken per minute \\
\hline$d_{\text {syst }}$ & $\begin{array}{l}\text { Dead space volume of the } \\
\quad \text { apparatus used (L) }\end{array}$ & $\begin{array}{l}\text { Volume of air that remains within the CPET apparatus e.g. breathing valve, connectors and } \\
\text { mouth piece exterior to the mouth }\end{array}$ \\
\hline \multirow[t]{2}{*}{$V_{\text {eqCo }}$} & $\begin{array}{l}\text { Ventilatory equivalent for carbon } \\
\text { dioxide }\end{array}$ & Measures the efficiency of ventilation \\
\hline & & Equation $\mathrm{B}: V_{\mathrm{eqCO}_{2}}=\frac{V_{\mathrm{E}}^{\prime}-\left(\mathrm{d}_{\mathrm{syst}} \times \mathrm{RR}\right)}{V_{\mathrm{CO}_{2}}^{\prime}}$ \\
\hline
\end{tabular}


increase in $V^{\prime} \mathrm{CO}_{2}$. An increase in $\mathrm{CO}_{2}$ concentration in the blood stimulates chemoreceptors, leading to a proportional increase in the $V_{E}^{\prime}$ to remove the excess $\mathrm{CO}_{2}$ produced. Therefore, there is a linear positive relationship between $V^{\prime} \mathrm{CO}_{2}$ and $V_{\mathrm{E}}^{\prime}$ as both increase in proportion to one another. The initial slope of this graph can be described by equation A [26] in table 1 . A high dead space volume $\left(V_{\mathrm{D}}\right) / V_{\mathrm{T}}$ will result in an increased gradient of the $V_{\mathrm{E}}^{\prime} / V^{\prime} \mathrm{CO}_{2}$ slope. This is because there is a decreased proportion of air undergoing gas exchange during ventilation, which can be caused by a variety of pathologies. This means that a higher $V^{\prime}$ E will be required to remove a set volume of $\mathrm{CO}_{2}$ produced.

The respiratory compensation point is the point at which acidosis becomes the predominant stimulus for ventilation [26]. This reflects the point during exercise at which the circulatory system can no longer buffer the increased acid produced during anaerobic respiration. At this point the gradient of the slope increases. This is because the $V_{\mathrm{CO}_{2}}^{\prime}$ does not change significantly here. However, the $V_{\mathrm{E}}^{\prime}$ significantly increases due to the new acidic stimulus acting on chemoreceptors.

The second parameter involving $V_{\mathrm{E}}^{\prime}$ and $V_{\mathrm{CO}_{2}}^{\prime}$ is the $V_{\mathrm{eqCO}}$. Changes in $V_{\mathrm{eqCO}}$ are shown by plotting $V_{\text {eqCO }}$ on the y-axis against time or work rate on the x-axis. The parameter is related to equation B in table 1. The differences between the $V_{\mathrm{E}}^{\prime} / V^{\prime} \mathrm{CO}_{2}$ slope and the $V_{\mathrm{eq} \mathrm{CO}_{2}}$ will be highlighted by discussing how the $V_{\text {eqCO }}$ changes with light exercise. This is different compared with the $V^{\prime}{ }_{\mathrm{E}} / V^{\prime} \mathrm{CO}_{2}$ slope.

At low workloads of exercise, both $V_{\mathrm{CO}_{2}}^{\prime}$ and $V_{\mathrm{E}}^{\prime}$ are increasing, but the value of the $V_{\mathrm{eqCO}}$ decreases. This is because $V_{\text {eqCO}}$ reflects the changes in the volume of gas now required to remove a set volume of $\mathrm{CO}_{2}$ produced from exercise. It is an indication of how efficient the lungs are. As light exercise commences (e.g. producing $50 \mathrm{~W}$ ), the ventilation-perfusion ratio in the apex of the lungs improves [27] and a larger proportion of air drawn in to the lungs is able to undergo gas exchange (a decrease in the $\mathrm{d} / V_{\mathrm{T}}$ ). This increased efficiency means that for the increase in $V_{\mathrm{CO}_{2}}$ that occurs with exercise, a proportionally smaller increase in ventilation is now required to maintain a normal arterial $\mathrm{CO}_{2}$ tension $\left(P_{\mathrm{aCO}}\right)$. This is mathematically illustrated by equation $\mathrm{B}$ in table 1 . Incorporating the system dead space volume directly into equation $\mathrm{B}$ allows the changes in ventilation-perfusion ratios of the lung described to directly influence the value for $V_{\text {eq }} \mathrm{CO}_{2}$. This allows the $V_{\mathrm{eqCO}}$ to be an accurate parameter of how efficient the lungs are at different times during the exercise test. Above all, the equation demonstrates that $V_{\mathrm{eqCO}}$ is not the same parameter as the $V^{\prime}{ }_{E} / V^{\prime} \mathrm{CO}_{2}$ slope.

As the exercise intensity increases, the value of the $V_{\mathrm{eqCO}}$ stops decreasing and tapers off as the lungs reach their most efficient ventilatory state. However, once the respiratory compensation point is reached, the $V_{\mathrm{eqCO}}$ then increases like the $V_{\mathrm{E}}^{\prime} / V^{\prime} \mathrm{CO}_{2}$ slope due to the disproportionate increase in $V_{\mathrm{E}}^{\prime}$ compared to $V^{\prime} \mathrm{CO}_{2}$, as a result of acidaemia.

The $V_{\mathrm{eqCO}}$ will be the focus of this review, given that the research demonstrates that this parameter is most useful in the diagnosis of dysfunctional breathing.

\section{Changes in $\mathrm{V}_{\text {eqCO }}$ in patients with dysfunctional breathing}

Given the subtypes of dysfunctional breathing have been described above, it is important to characterise what types of dysfunctional breathing patients have been included in experiments that will be analysed. Past research has been directed towards changes in $V_{\text {eqCO }}$ in patients who have hyperventilation syndrome (HVS). Therefore, changes in $V_{\mathrm{eqCO}_{2}}$ will be described in dysfunctional breathing patients who fall into the HVS category [4].

It should be noted that individuals who suffer with HVS will be discussed in two separate groups. The first group include those who have a resting respiratory alkalosis pattern on an arterial blood gas (ABG) test before CPET. The second group includes those who have an unremarkable pattern (normocapnic, normal $\mathrm{pH}$ ) on an ABG test at rest before CPET. A distinction is being made here because results show hypocapnic patients at the start of testing will give different $V_{\text {eqCO}}$ results compared to those patients who are normocapnic before testing. All key results of the studies described have been outlined in table 2 .

Individuals with HVS that hyperventilate at rest before CPET have been characterised by KINNULA and Sovijarvi [28]. 10 individuals suspected of HVS who were absent of diagnosed medical conditions underwent CPET. The patients were included based on a respiratory alkalosis at rest (table 2).

Despite this respiratory alkalosis recorded at rest, it should be noted that these patients cannot be said to be chronically hyperventilating purely based on their ABG. This is because their hyperventilation could have been induced by anxiety before exercise testing. Whilst a stress and anxiety assessment was not used in 
TABLE 2 Relevant data from studies used to demonstrate the reviews conclusions

\begin{tabular}{|c|c|c|c|}
\hline First author [ref.] and parameter & HVS suspects & Controls & p-value \\
\hline KINNULA [28] & $n=10$ & $n=10$ & \\
\hline Resting $P_{\mathrm{aCO}_{2}} \mathrm{kPa}$ & $4.10 \pm 0.60$ & ND & \\
\hline Resting pH & $7.46 \pm 0.06$ & ND & \\
\hline$V_{\text {eqCO }}$ at $40-50 \mathrm{~W}$ exercise & $41.8 \pm 6.00$ & $30.0 \pm 3.30$ & 0.01 \\
\hline Jack [29] & $n=23$ & $n=23$ & \\
\hline Resting $P_{\mathrm{aCO}_{2}} \mathrm{kPa}$ & $3.87 \pm 0.60$ & ND & \\
\hline$V_{\text {eqCO }}$ at $40 \mathrm{~W}$ exercise & $38.4 \pm 5.70$ & $28.9 \pm 2.50$ & ND \\
\hline BRAt [19] & $n=29$ & $n=29$ & \\
\hline Resting arterial $P_{\mathrm{aCO}_{2}} \mathrm{kPa}$ & $4.80(4.40-4.93)$ & $4.80(4.53-5.07)$ & 0.44 \\
\hline Resting arterial $\mathrm{pH}$ & $7.45(7.43-7.47)$ & $7.44(7.43-7.45)$ & 0.07 \\
\hline Peak exercise $P_{\mathrm{aCO}_{2}} \mathrm{kPa}$ & $3.87 \pm 0.53$ & $4.67 \pm 0.40$ & $<0.01$ \\
\hline Peak exercise arterial $\mathrm{pH}$ & $7.47(7.46-7.50)$ & $7.40(7.37-7.42)$ & $<0.01$ \\
\hline Resting $\mathrm{d} / V_{\mathrm{T}}$ & $0.21 \pm 0.11$ & $0.22 \pm 0.10$ & 0.77 \\
\hline Peak exercise $d / V_{T}$ & $0.18 \pm 0.05$ & $0.13 \pm 0.06$ & $<0.01$ \\
\hline$V_{\mathrm{eqCO}_{2}}$ at rest & $38.0(33.0-44.0)$ & $37.0(32.0-40.0)$ & 0.31 \\
\hline$V_{\text {eqCO }}$ at peak exercise & $38.0(35.0-43.0)$ & $31.0(27.0-34.0)$ & $<0.01$ \\
\hline$V_{\mathrm{E}}^{\prime} / V^{\prime} \mathrm{CO}_{2}$ slope & $37.0(33.0-34.0)$ & $27.0(24.0-30.0)$ & $<0.01$ \\
\hline TROOSTERS [30] & $n=24$ & $n=20$ & \\
\hline NQ scores & $30.3 \pm 9.70$ & $7.90 \pm 5.20$ & $<0.001$ \\
\hline
\end{tabular}

Data are presented as mean \pm SD or median (interquartile range), unless otherwise stated. HVS: hyperventilation syndrome; $P_{\mathrm{aCO}_{2}}$ : arterial carbon dioxide tension; $V_{\mathrm{eqCO}}$ : ventilatory equivalent for carbon dioxide; $d / \mathrm{V}_{\mathrm{T}}$ : ratio of dead space volume to tidal volume; ND: not disclosed in study. $V^{\prime}{ }_{E} / V^{\prime} \mathrm{CO}_{2}$ slope: gradient of the $V_{E}^{\prime}$ (abscissa) and $V^{\prime} \mathrm{CO}_{2}$ (ordinate) slope; NQ: Nijmegen Questionnaire.

this study, other studies have shown that HVS patients score significantly above average on Hospital Anxiety and Depression Scale scores [29]. The base excesses for the 10 HVS suspects were also normal, which further suggests that these HVS suspects were not chronically hyperventilating in day-to-day life. This is because a metabolic compensation, reflected by a decrease in the base excess, would be expected in individuals who are chronically hypocapnic due to chronic hyperventilation [31]. This highlights the importance not to separate patients based on whether they are chronically or intermittently hyperventilating, alternatively basing categorisation on whether they are normocapnic or hypocapnic before the exercise test. However, it should be noted that other physiological parameters outside the scope of this review, such as respiratory exchange ratio, can indicate how acute any hyperventilation may be.

When these patients were subjected to exercise testing, their $V_{\mathrm{eqCO}}$ changed in a similar pattern to the controls. This included a decrease in $V_{\text {eqCO}}$ as exercise intensity increased, followed by a subsequent increase in $V_{\mathrm{eqCO}}$ at maximal work load. The difference between the two groups was that the $V_{\text {eqCO }}$ was significantly higher at most exercise loads in the suspected HVS patients compared to the controls during the test. The key aspect identified by Kinnula and SoviJarvi [28] was that $V_{\mathrm{eqCO}}$ was significantly raised compared to controls at a low intensity of exercise (table 2). These findings suggest that $V_{\text {eqCO }}$ is significantly elevated in early stages of exercise for individuals who have suspected HVS and commence the exercise testing hypocapnic.

This finding is supported by experiments carried out by JACK et al. [29]. 39 individuals with suspected HVS (described as idiopathic hyperventilation in this study) were included by demonstrating these suspects had arterial hypocapnia at rest (table 2) and were free of other organic diseases. The individuals with arterial hypocapnia before testing also had a significantly higher $V_{\mathrm{eqCO}}$ than controls at an exercise intensity of $40 \mathrm{~W}$ (table 2), supporting the findings by KinNula and SoviJARVI [28].

The cause for these differences between controls and HVS suspects have been discussed in both studies. If an individual starts with a lower $P_{\mathrm{aCO}}$, then using the equations described in table 1 , it can be predicted that they will have a decreased value of $V^{\prime} \mathrm{CO}_{2}$ during exercise compared to controls. This difference can cause an increased $V_{\text {eqCO}}$ in the individuals who start hypocapnic at all exercise intensities. Furthermore, Kinnula and SoviJarvi [28] demonstrated a negative correlation of $\mathrm{r}=-0.77(\mathrm{p}<0.01)$ exists between the $P_{\mathrm{aCO}_{2}}$ of an individual and the $V_{\mathrm{eqCO}}$ at light exercise in this study. This suggests that the elevated $V_{\mathrm{eqCO}}$ seen in individuals with suspected HVS, who are hypocapnic when starting testing, is predominantly attributable to consistently different $P_{\mathrm{aCO}}$ levels between the controls and HVS suspects. This is supported 
by JACK et al. [29], who use the equations shown in table 1 to demonstrate how differences in $V_{\text {eqCO }}$ in their study can be solely attributed to changes in $P_{\mathrm{aCO}}$ between the two groups they studied.

Another study has been undertaken by BRAT et al. [19]. This included 29 individuals suspected of having HVS who had documented dyspnoea and periods of respiratory alkalosis. They also did not have any other medical conditions. Importantly, these individuals were different from the studies cited above given the ABG samples of the suspected HVS patients at rest were not significantly different from healthy controls (table 2). This study used CPET as well and its results broadly align with the other studies cited [28, 29]: $V_{\mathrm{eqCO}}$ is raised in patients with suspected HVS during low levels of exercise compared to controls (table 2). This finding is also supported by an elevated $V^{\prime}{ }_{E} / V^{\prime} \mathrm{CO}_{2}$ slope in the subjects suspected of HVS, when compared to controls.

However, there are some key differences in the data presented between Brat et al. [19] and the other studies cited above [28, 29] These differences can be linked to the variations in pre-test $P_{\mathrm{aCO}_{2}}$ of the subjects used in each of the studies. The study carried out by Brat et al. [19] showed that the $V_{\mathrm{eqCO}}$ ratio of the HVS suspects did not change with work load during exercise when compared to the controls (figure 1).

The $P_{\mathrm{aCO}_{2}}$ of HVS suspects that started normocapnic decreased their $\mathrm{Pa}_{\mathrm{CO} 2}$ as exercise work load increased (table 2) [19]. Hyperventilation during CPET causes a disproportionate decrease in $P_{\mathrm{aCO}}$, rather than maintaining $P_{\mathrm{aCO}}$ like controls do. This counters the decreases in $\mathrm{d} / V_{\mathrm{T}}$ with increased exercise intensity, which leads to the $V_{\mathrm{eqCO}}$ staying constant with exercise work load. Furthermore, BRAT et al. [19] show that the $\mathrm{d} / V_{\mathrm{T}}$ in HVS suspects does not decrease with exercise as much as in controls (table 2). This could also be due to the onset of hyperventilation in the HVS suspects during the exercise. Hyperventilation could lead to inefficient breathing mechanics, thus not allowing maximal gas exchange within the lungs. This

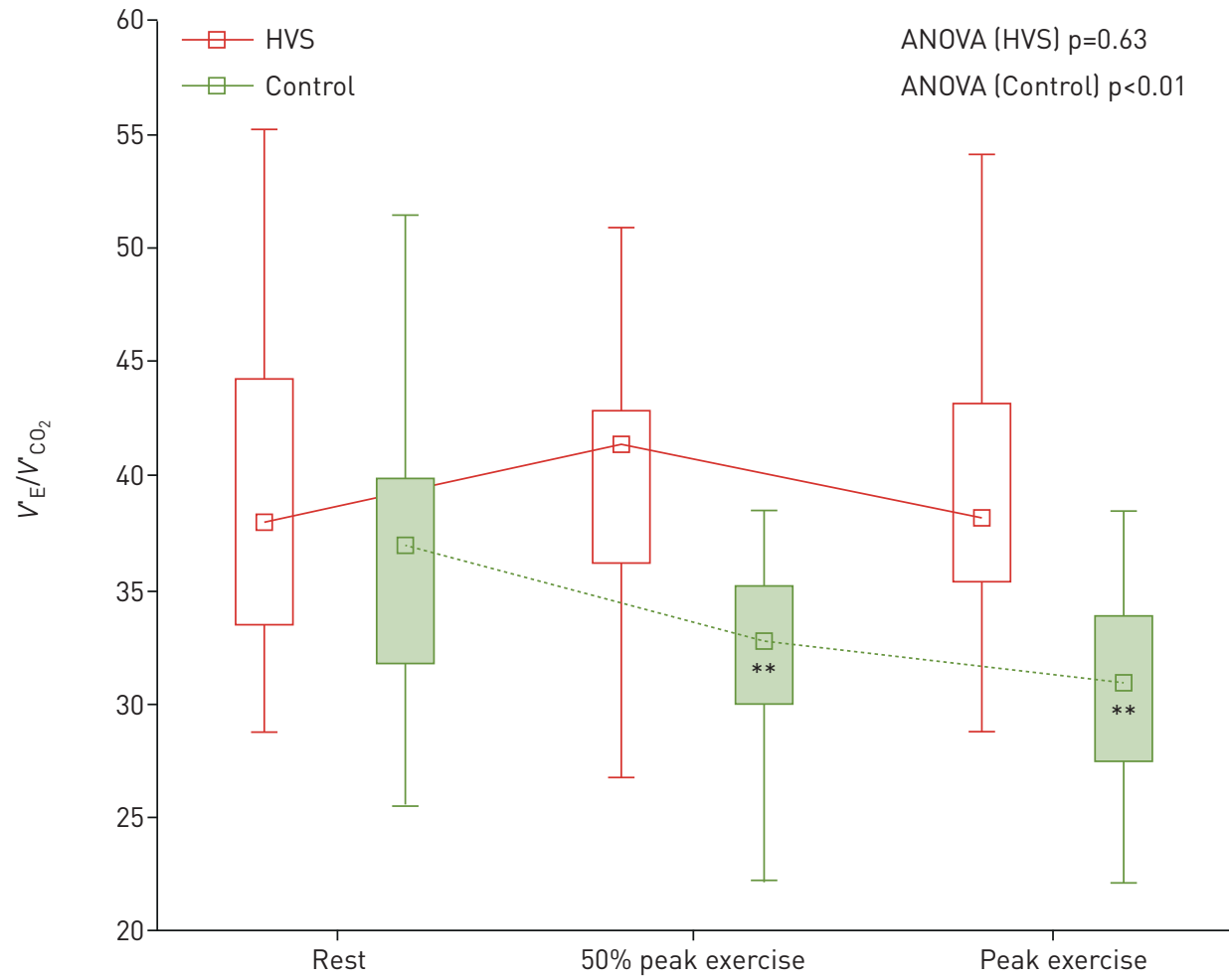

FIGURE 1 Graph illustrating the changes in ventilatory equivalent for carbon dioxide (described as $V^{\prime}{ }_{E} / V^{\prime} \mathrm{CO}_{2}$ in this study) in hyperventilation syndrome (HVS) suspects who are normocapnic before commencing exercise testing compared to controls. ANOVA values were used to determine significant changes in ventilatory equivalent for carbon dioxide within the groups as exercise intensity increased. ${ }^{\star \star}: p<0.01$ compared with ventilatory equivalent for carbon dioxide at rest. $V_{\mathrm{E}}^{\prime}$ : minute ventilation; $V^{\prime} \mathrm{CO}_{2}$ : carbon dioxide production. Reproduced from [19] with permission from the publisher. 
would result in a smaller decrease in $d / V_{\mathrm{T}}$ with exercise in the HVS suspects compared to controls (table 2).

Troosters et al. [30] have also undertaken experiments to characterise exercise in individuals suspected of HVS. HVS suspects were included based on the absence of organic disease, whilst scoring highly on the Nijmegen Questionnaire (table 2). It was unsure whether these individuals started the CPET testing hypocapnic or normocapnic. This study found no significant difference between HVS suspects and controls when measuring the $V_{\mathrm{eqCO}}$. The study did not disclose values of $V_{\mathrm{eqCO}_{2}}$ for either group.

However, the study did cite a correlation between the severity of the symptoms of a patient (documented by whether they were on sick leave or not) and $V_{\mathrm{eqCO}}$. Individuals who were on sick leave had a higher $V_{\text {eqCO }}$ than other HVS suspects. This suggests that there could be a correlation between the severity of symptoms HVS suspects feel and $V_{\text {eq }} \mathrm{CO}_{2}$. The difference in results from this study to the others cited could be due to a wide range of severities of the HVS patients. This large variation in severity within the experimental group is reflected by a large standard deviation of the Nijmegen Questionnaire scores (table 2).

Overall, studies that characterise the changes seen in $V_{\mathrm{eqCO}_{2}}$ in patients with suspected HVS have been described. In all studies cited, the individuals included were free of organic disease. Therefore, the conclusions made here may only apply to individuals free of other cardiopulmonary co-morbidities. In conclusion, there is a consistent elevation of $V_{\mathrm{eqCO}}$ during light exercise in patients with $\mathrm{HVS}$, with no difference being described between males and females. It must be noted how the data shows that $V_{\mathrm{eqCO}}$ may or may not change with exercise intensity, dependent on the starting $P_{\mathrm{aCO}_{2}}$ of the patient (and whether it subsequently changes during testing). Given significant differences in $V_{\mathrm{eqCO}}$ have been described in individuals with HVS, the reliability of using this physiological parameter to help diagnose dysfunctional breathing must now be discussed.

Reliability of $\mathrm{V}_{\text {eqCO }}$ as a physiological indicator for dysfunctional breathing

A significant difference in $V_{\text {eqCO }}$ has been shown in individuals with HVS. However, to effectively use $V_{\text {eq } \mathrm{CO}_{2}}$ in an algorithm to diagnose dysfunctional breathing, recommended uses of values must be proposed. Other conditions that can present with an elevated $V_{\text {eqCO}}$ during exercise and breathlessness must also be mentioned.

Kinnula and Sovisarvi [28] propose using a value of 35 as a cut off for the $V_{\mathrm{eq} \mathrm{CO}_{2}}$ at light exercise $(40-50 \mathrm{~W})$. This value would have separated all HVS suspects from normal controls at this exercise intensity, apart from one (sensitivity: 91\%, specificity: 100\%). This value of 35 sits within the means/ medians for HVS suspects of all other studies quoted (table 2). However, $V_{\mathrm{eqCO}}$ only characterises one aspect of dysfunctional breathing and, therefore, needs to be used within a diagnostic algorithm. Thus, it is not appropriate to talk about using $V_{\mathrm{eqCO}}$ as a single parameter. The sensitivities and specificities quoted in one study have been included here to demonstrate that it is a reliable indicator of physiological abnormalities in dysfunctional breathing. Its use in an algorithm will be discussed later.

Heart failure and pulmonary hypertension patients can also present with a raised $V_{\text {eq }} \mathrm{CO}_{2}$ during intense exercise [32, 33]. Therefore, patients presenting with breathlessness and a raised $V_{\mathrm{eqCO}}$ should have these diagnoses ruled out using other diagnostic tools specific for that condition.

The advantages and drawbacks of using $\mathrm{V}_{\text {eqCO}}$ in a diagnostic algorithm for dysfunctional breathing Given dysfunctional breathing is a multi-faceted conditions and $V_{\mathrm{eqCO}}$ only describes one parameter measured during CPET, $V_{\mathrm{eqCO}}$ must be used in a diagnostic algorithm to diagnose dysfunctional breathing. A proposed diagnostic algorithm has been described (figure 2). The advantages of using $V_{\mathrm{eqCO}_{2}}$ in a diagnostic algorithm include having an objective value to aid the diagnosis of dysfunctional breathing and to direct patients to certain treatment strategies. The use of CPET can also be part of the treatment for certain types of dysfunctional breathing.

Using 35 as a diagnostic cut off value for $V_{\text {eqCO }}$ has been outlined above. Using this value allows an objective diagnosis of a physiological abnormality in dysfunctional breathing patients. This value needs to be verified in larger sample sizes of patients. However, it would allow for a more consistent diagnosis of dysfunctional breathing with physiological abnormalities.

$V_{\text {eqCO }}$ can also direct patient treatment. A $V_{\text {eqCO }}$ above 35 suggests that patients either chronically or intermittently hyperventilate. This can lead to hypocapnia. JoHANSEN et al. [34] have described a study that 


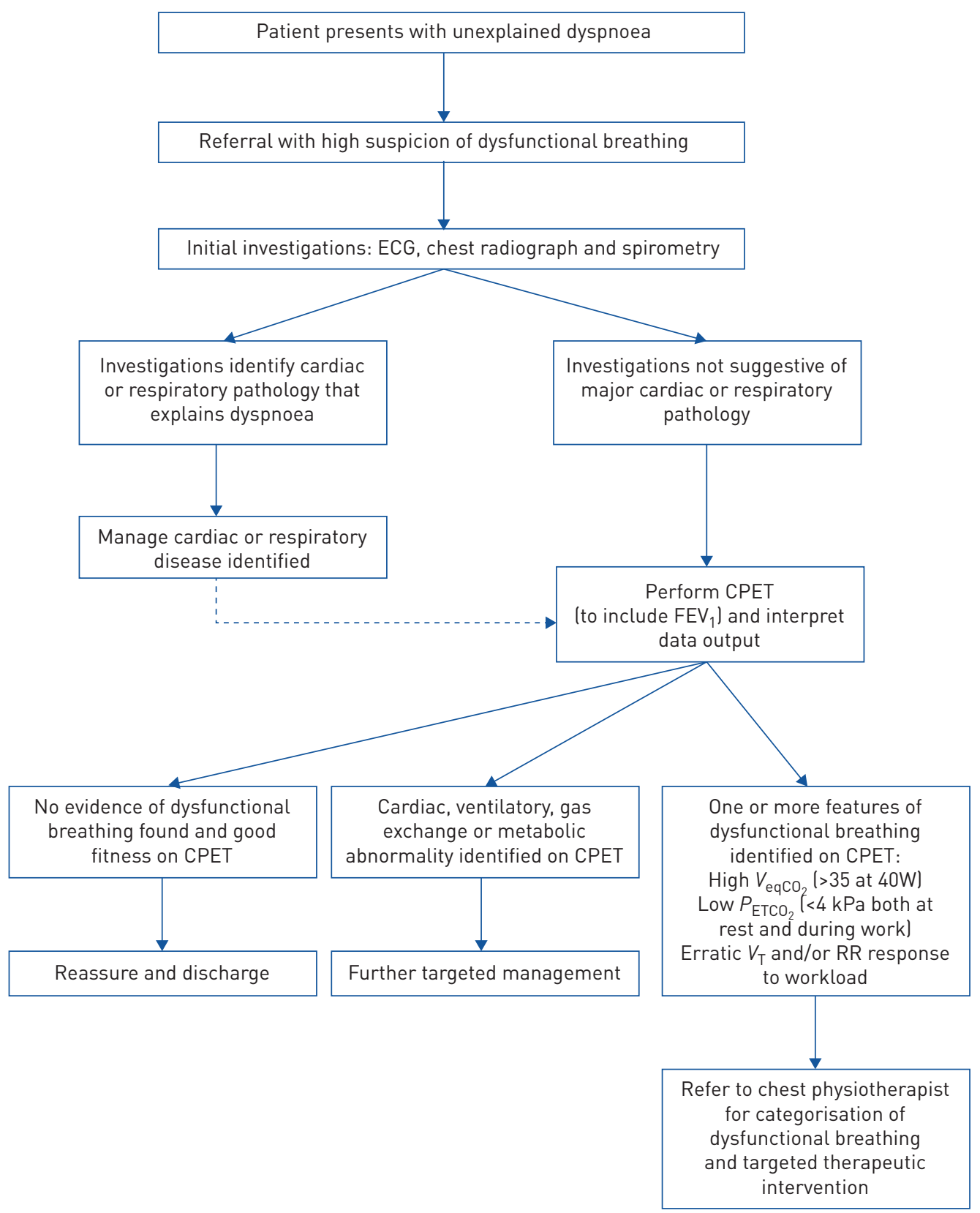

FIGURE 2 A proposed clinical pathway for patients suspected of dysfunctional breathing, based on current practice at the Cambridge University Hospital trust. Values quoted are based on data from cited studies [19, 28, 29]. $\mathrm{FEV}_{1}$ : forced expiratory volume in $1 \mathrm{~s} ; V_{\text {eqCO }}$ : ventilatory equivalent for $\mathrm{CO}_{2} ; P_{\mathrm{ETCO}_{2}}$ : end-tidal carbon dioxide tension; $V_{\mathrm{T}}$ : tidal volume; RR: respiratory rate.

uses a partial rebreathing mask to induce normocapnia in individuals who are chronically hypocapnic (capillary $\mathrm{CO}_{2}$ tension $<4.7 \mathrm{kPa}$ ). The study was only conducted on six patients who were chronically hypocapnic. However, using the mask for $2 \mathrm{~h}$ per day for 4 weeks led to a reduction in daily symptoms (change in Nijmegen Questionnaire score $=-3.8$; $\mathrm{p}=0.046$ ) and raised capillary $\mathrm{CO}_{2}$ tension significantly back to normocapnic levels ( $+0.45 \mathrm{kPa}$; $\mathrm{p}=0.046$ ). More work needs to be done to characterise whether this technique could aid individuals who intermittently hyperventilate and have fluctuating $P_{\mathrm{acO}}$ values. However, the use of $V_{\mathrm{eqCO}}$ can give clinicians an idea as to which patients could benefit from such treatments.

Undertaking CPET testing in individuals with dysfunctional breathing can also be a therapy in itself. Individuals suffering from certain types of dysfunctional breathing have been shown to score higher on 
Hospital Anxiety and Depression Scores [29]. Thus, undertaking testing on these individuals can give two possible outcomes. CPET could reveal a normal $V_{\mathrm{eqCO}}$. This can be reassuring to patients, which can alleviate anxiety. On the other hand, the $V_{\mathrm{eqCO}_{2}}$ could be raised indicating a physiological abnormality, thus directing appropriate treatment [34].

Using CPET in the diagnosis of dysfunctional breathing does have its drawbacks. Given individuals are only on the exercise bike for $18 \mathrm{~min}$, this may not be a long enough time to capture abnormalities in ventilation. Thus, individuals who do experience intermittent hyperventilation may not hyperventilate during the test, leading to a false-negative result. It also cannot be ignored that many studies cited here have small $(<29)$ sample sizes [19, 28-30, 34]. This indicates a need to undertake further research in this area to confirm the findings discussed above. The use of $V_{\mathrm{eqCO}}$ has also only been characterised in HVS. Other subtypes need to be studied using this physiological indicator to see whether CPET, and specifically $V_{\text {eqCO}}$, can be used to aid the diagnosis of these sub-types of dysfunctional breathing as well. $V_{\text {eqCO }}$ is also not the only physiological measure of an individual's breathing produced by CPET. Patients with dysfunctional breathing have also been shown to have raised respiratory rates, decreased end-tidal carbon dioxide tension and decreased $V_{\mathrm{T}}$ [19]. Using an exact cut off point for $V_{\mathrm{eqCO}_{2}}$ may leave clinicians in a dilemma when patients have a $V_{\mathrm{eqCO}}<35$, but clearly show abnormal physiological breathing patterns when breathing frequency and $V_{\mathrm{T}}$ are observed for example. Discussing the other physiological parameters CPET shows about a subject's breathing is out of the scope of this review, but $V_{\text {eqCO }}$ should be used in conjunction with other physiological parameters CPET can provide.

This review supports the use of an objective cut off for the $V_{\mathrm{eqCO}}$ used in the diagnosis of dysfunctional breathing. However, more work must be done to understand how other physiological variables could be used alongside $V_{\text {eqCO }}$ to aid the diagnosis of dysfunctional breathing. Furthermore, the prognostic value of $V_{\text {eqCO }}$ must also be determined when more data has been collected and the above diagnostic algorithm has been verified in clinical practice. $V_{\mathrm{eqCO}}$ can be used as an accurate prognostic factor in pulmonary hypertension and heart failure $[35,36]$. However, no data currently exists that suggests $V_{\mathrm{eqCO}_{2}}$ can or cannot be used as a prognostic indicator for patients with dysfunctional breathing (including HVS).

\section{Conclusion}

This review supports the use of $V_{\mathrm{eqCO}}$ in a diagnostic algorithm for dysfunctional breathing. A $V_{\mathrm{eqCO}}>35$ gives an objective measure as to whether an individual has a physiological abnormality in their breathing pattern. However, more research needs to be undertaken to understand the diagnostic role of $V_{\text {eqCO }}$ in other subtypes of dysfunctional breathing, including individuals with other cardiopulmonary co-morbidities. The understanding of how the $V_{\mathrm{eqCO}_{2}}$ can be used with other physiological parameters and assessments of dysfunctional breathing must also be further researched.

Previous articles in this series: No. 1: Laveneziana P, Di Paolo M, Palange P. The clinical value of cardiopulmonary exercise testing in the modern era. Eur Respir Rev 2021; 30: 200187. No. 2: Agnostoni P, Sciomer S, Palermo P, et al. Minute ventilation/carbon dioxide production in chronic heart failure. Eur Respir Rev 2021; 30: 200141.

Provenance: Commissioned article, peer reviewed.

Conflict of interest: None declared.

\section{References}

1 Chenivesse C, Similowski T, Bautin N, et al. Severely impaired health-related quality of life in chronic hyperventilation patients: exploratory data. Respir Med 2014; 108: 517-523.

2 Hagman C, Janson C, Emtner M. Breathing retraining: a five-year follow-up of patients with dysfunctional breathing. Respir Med 2011; 105: 1153-1159.

3 Thomas M, McKinley RK, Freeman E, et al. The prevalence of dysfunctional breathing in adults in the community with and without asthma. Prim Care Respir J 2005; 14: 78-82.

4 Boulding R, Stacey R, Niven R, et al. Dysfunctional breathing: a review of the literature and proposal for classification. Eur Respir Rev 2016; 25: 287-294.

5 Barker N, Everard ML. Getting to grips with "dysfunctional breathing”. Paediatr Respir Rev 2015; 16: 53-61.

6 Vidotto LS, de Carvalho CRF, Harvey A, et al. Dysfunctional breathing: what do we know? J Bras Pneumol 2019; 45: e20170347.

7 Sun X-G, Hansen JE, Garatachea N, et al. Ventilatory efficiency during exercise in healthy subjects. Am J Respir Crit Care Med 2002; 166: 1443-1448.

8 Courtney R. The functions of breathing and its dysfunctions and their relationship to breathing therapy. Int $J$ Osteopath Med 2009; 12: 78-85. 
9 Depiazzi J, Everard ML. Dysfunctional breathing and reaching one's physiological limit as causes of exercise-induced dyspnoea. Breathe 2016; 12: 120-129.

10 Prys-Picard CO, Kellett F, Niven RM. Disproportionate breathlessness associated with deep sighing breathing in a patient presenting with difficult-to-treat asthma. Chest 2006; 130: 1723-1725.

11 Courtney R, Greenwood KM, Cohen M. Relationships between measures of dysfunctional breathing in a population with concerns about their breathing. J Bodyw Mov Ther 2011; 15: 24-34.

12 Todd S, Walsted ES, Grillo L, et al. Novel assessment tool to detect breathing pattern disorder in patients with refractory asthma. Respirology 2018; 23: 284-290.

13 Mitchell A, Bacon C, Moran R. Reliability and Determinants of Self-Evaluation of Breathing Questionnaire (SEBQ) Score: a symptoms-based measure of dysfunctional breathing. Appl Psychophysiol Biofeedback 2015; 41: 111-120.

14 van Dixhoorn J, Duivenvoorden HJ. Efficacy of Nijmegen Questionnaire in recognition of the hyperventilation syndrome. J Psychosom Res 1985; 29: 199-206.

15 van Dixhoorn J, Folgering $\mathrm{H}$. The Nijmegen Questionnaire and dysfunctional breathing. ERJ Open Res 2015; 1 00001-2015.

16 Stanton AE, Vaughn P, Carter R, et al. An observational investigation of dysfunctional breathing and breathing control therapy in a problem asthma clinic. J Asthma 2008; 45: 758-765.

17 Courtney R, van Dixhoorn J, Cohen M. Evaluation of breathing pattern: comparison of a Manual Assessment of Respiratory Motion (MARM) and respiratory induction plethysmography. Appl Psychophysiol Biofeedback 2008; 33: 91-100.

18 Levai IK, Massaroni C, Hull JH, et al. Optoelectronic plethysmography (OEP) in the assessment of dysfunctional breathing (DB) in athletes. Eur Respir J 2016; 48, Suppl. 60: PA2248.

19 Brat K, Stastna N, Merta Z, et al. Cardiopulmonary exercise testing for identification of patients with hyperventilation syndrome. PLoS One 2019; 14: e0215997.

20 Degani Costa LH, Rodrigues MT, Fonseca AXC, et al. The role of cardiopulmonary exercise testing in the diagnosis of psychogenic dyspnoea. Eur Respir J 2016; 48, Suppl. 60: OA2016.

21 Griffiths O, Anindo B. Cardiopulmonary exercise testing (CPET) in patients with unexplained dyspnoea. Eur Respir J 2014; 44, Suppl. 58: P4886.

22 Jayadev A, Devani N, Geddes L, et al. The role of cardiopulmonary exercise testing in the investigation of complex unexplained breathlessness. Eur Respir J 2017; 50, Suppl. 61: PA4538.

23 Roberts S, Macdonald W, Townson M, et al. Cardiopulmonary excercise testing in the diagnosis of chronic unexplained dyspnoea. Eur Respir J 2017; 50, Suppl. 61: PA4537.

24 Thing JER, Mukherjee B, Murphy K, et al. P189 Evaluation of the role of cardio-pulmonary exercise testing in the diagnosis of unexplained breathlessness. Thorax 2011; 66, Suppl. 4: A144 LP-A145.

25 Wasserman K. Diagnosing cardiovascular and lung pathophysiology from exercise gas exchange. Chest 1997; 112: 1091-1101.

26 Mezzani A. Cardiopulmonary Exercise Testing: Basics of Methodology and Measurements. Ann Am Thorac Soc 2017; 14, Suppl. 1: S3-11.

27 Harf A, Pratt T, Hughes JM. Regional distribution of VA/Q in man at rest and with exercise measured with krypton-81 m. J Appl Physiol Respir Environ Exerc Physiol 1978; 44: 115-123.

28 Kinnula VL, Sovijarvi AR. Elevated ventilatory equivalents during exercise in patients with hyperventilation syndrome. Respiration 1993; 60: 273-278.

29 Jack S, Rossiter HB, Warburton CJ, et al. Behavioral influences and physiological indices of ventilatory control in subjects with idiopathic hyperventilation. Behav Modif 2003; 27: 637-652.

30 Troosters T, Verstraete A, Ramon K, et al. Physical performance of patients with numerous psychosomatic complaints suggestive of hyperventilation. Eur Respir J 1999; 14: 1314-1319.

31 Berend K. Diagnostic use of base excess in acid-base disorders. N Engl J Med 2018; 379: 496.

32 Deboeck G, Niset G, Lamotte $\mathrm{M}$, et al. Exercise testing in pulmonary arterial hypertension and in chronic heart failure. Eur Respir J 2004; 23: 747-751.

33 Clark AL, Poole-Wilson PA, Coats AJ. Relation between ventilation and carbon dioxide production in patients with chronic heart failure. J Am Coll Cardiol 1992; 20: 1326-1332.

34 Johansen $\mathrm{T}$, Jack $\mathrm{S}$, Dahl R. Normalizing $\mathrm{CO}_{2}$ in chronic hyperventilation by means of a novel breathing mask: a pilot study. Clin Respir J 2013; 7: 359-366.

35 Myers J, Arena R, Oliveira RB, et al. The lowest $V_{\mathrm{E}} / V_{\mathrm{CO} 2}$ ratio during exercise as a predictor of outcomes in patients with heart failure. J Card Fail 2009; 15: 756-762.

36 Schwaiblmair M, Faul C, von Scheidt W, et al. Ventilatory efficiency testing as prognostic value in patients with pulmonary hypertension. BMC Pulm Med 2012; 12: 23. 